\title{
EXPECTATIVAS RACIONAIS COM INFORMAÇĀO ASSIMÉTRICA: UMA INTRODUÇÃO
}

\section{Paulo Cesar Coutinho*}

\section{Resumo}

Esse artigo é uma introdução à teoria de equilíbrio com expectativas racionais. Cobrimos os casos com informação simétrica e com informação assimétrica. Mostramos que com mercados complexos e um número finito de estados da natureza existe sempre equilíbrio com expectativas racionais Pareto-ótimo. Todavia podem existir outros equilíbrios com expectativas racionais Pareto dominados.

\section{Abstract}

This is an introduction to the theory of rational expectations. We cover the case of symmetric information and the case of asymmetric information. We show that with complete markets and a finite number of states there always exists a Pareto optimum rational expectations equilibrium. However, it is possible under the same circumstances to have other rational expectations equilibria that are Pareto dominated.

\section{Introdução.}

Este artigo é parte de um projeto maior onde pretendemos fazer um relato dos principais resultados da teoria econômica da incerteza e informação, de forma rigorosa mas sem utilização de métodos avançados de matemática. Neste artigo, cobrimos a teoria de equilíbrio competitivo em economias estocásticas com informação assimétrica. Todavia o campo ainda é vasto demais para apenas um artigo. Restringimos-nos ao estudo do principal conceito de equilíbrio que emerge nessas economias que é o de equilibrio com expectativas racionais (EER). Na seção 2, estudaremos a origem do conceito de expectativas racionais e sua transformação em conceito de equilíbrio para economias estocásticas. Na seção 3 , introduziremos assimetria de informação e estudaremos como o conceito de equilíbrio com expectativas racionais permite que se modele economias sujeitas à incerteza e informação assimétrica e como, pela primeira vez, podemos estudar de forma rigorosa à idéia de que os preços agregam

*IMPA, Estrada Dona Castorina, 110, Rio de Janeiro, RJ 22460 Brasil

R. de Econometria

Rio de Janeiro

v. $13, \mathrm{n}$ 으 $1, \mathrm{pp} .79-104$

abril $1 \dot{993}$ 
informação dispersa na economia. $\mathrm{Na}$ seção 4, construiremos um modelo de equilíbrio geral para estudarmos a existência de EER e suas propriedades de bem-estar. Na seção 5 , construiremos exemplos de economias com mercados completos nos quais existe EER que não revela a informação e EER que não é Pareto Ótimo. Na seção 6, discutiremos brevemente a noção de EER em modelos macroeconômicos. $\mathrm{Na}$ seção 7, discutiremos outros conceitos de equilíbrios alternativos e alguns resultados adicionais.

2. Expectativas racionais como conceito de equilíbrio estocástico.

A idéia de expectativas racionais cresceu da insatisfação com modelos ad hoc de formação de expectativas em modelagens econométricas. Por exemplo, tornou-se prática por muitos anos (e ainda hoje é muito comum) supor que a taxa esperada de inflação é uma média ponderada das taxas de inflação prévias, como por exemplo o modelo de expectativas adaptivas de Nerlove (1958). Neste modelo, Nerlove sugere que a expectativa que um agente econômico no período $t$-1 forma a respeito do preço, no período $t, p_{t}^{e}$, satisfaz a equação

$$
p_{t}^{e}-p_{t-1}^{e}=\lambda\left(p_{t-1}-p_{t-1}^{e}\right)
$$

onde $p_{t-1}$ é o preço verdadeiro no período $t-1$ e $0<\lambda<1$. Isto é, o preço esperado no período $t$ é uma média ponderada entre o preço efetivamente realizado no período $t-1$ e o preço esperado no período $t-1$, com pesos iguais à $\lambda$ e $(1-\lambda)$. Substituindo $p_{t-1}^{e}$ na equação acima pela expressão

$$
p_{t-1}^{e}-p_{t-2}^{e}=\lambda\left(p_{t-2}-p_{t-2}^{e}\right)
$$

e procedendo assim sucessivamente, obtemos o modelo de defasagens distribuídas $p_{t}^{e}=\lambda \sum_{i=0}^{\infty}(1-\lambda)^{i} p_{t-i-1}$.

Como os estudos econométricos precisavam economizar em dados necessários para fazer a estimação, tornou-se essencial impor alguma restrição a priori na forma da estrutura de defasagem. Uma restrição sugerida por Muth (1960) era que a defasagem deveria ser racional. Com isto, Muth queria dizer que se a seqüência de preços 
$\left\{p_{t}\right\}$ é um processo estocástico, então o preço antecipado pelos agentes para o período $t$ calculado no período $t-1, p_{t}^{e}$ deveria ser dado pela fórmula $p_{t}^{e}=E\left[p_{t} \mid p_{t-1}, p_{t-2}, \ldots\right]$. Isto é, se o agente sabe a estrutura estocástica que gera os preços, então o preço que ele espera para o tempo $t$ é a esperança condicional de $p_{t}$, dadas as realizações anteriores do processo estocástico. Para ilustrar como a hipótese de Muth difere da hipótese de expectativas adaptivas, considere o seguinte exemplo. Suponhamos que o processo estocástico de geração de preços seja

$$
p_{t}=\left\{\begin{array}{l}
1, \text { se } t \leq 1 \\
2, \text { se } t \geq 2
\end{array}\right.
$$

Então num processo de expectativas adaptivas (ou de defasagens distribuídas), apenas no limite o preço estimado seria igual a $2, p_{2}^{e}=1$, $p_{3}^{e}=1(1-\lambda)+2 \lambda, \ldots, \lim _{t \rightarrow \infty} p_{t}^{e}=2$. Todavia, se o agente conhece o processo estocástico, com estrutura de defasagem racional $p_{t}^{e}=2$, $\forall t \geq 2$. O fato de que o modelo de expectativas racionais requer o conhecimento do processo estocástico pode ser abrandado pelo fato de que qualquer incerteza quanto ao verdadeiro processo estocástico pode ser modelado de um ponto de vista bayesiano.

Uma vez estabelecido o método de expectativas racionais, a pergunta seguinte é "de onde vem o processo estocástico?" Muth (1961) sugeriu que as prediçōes dos agentes econômicos devem ser as mesmas que as prediçōes feitas pela teoria econômica. Logo, o processo estocástico dos preços é aquele gerado pelos preços de equilíbrio quando os agentes têm expectativas racionais. Agora, expectativas racionais não é mais uma condiçâo de racionalidade dos agentes, mas uma noçâo de equilįrio para economias estocásticas.

Definição 1. (verbal) Um equilíbrio com expectativas racionais (EER) numa economia com incerteza é uma distribuição das alocaçōes e uma distribuição dos preços, tal que: 1) a distribuição das alocaçōes gera a distribuição dos preços e 2) a distribuição de alocaçōes é ótima para a distribuição dos preços.

Para clarificar as idéias, vamos reproduzir um modelo simples (Grossman 1975). Suponhamos que a demanda por um certo bem no 
tempo $t$ é dada pela equação ${ }^{1}$

$$
\tilde{p_{t}}=D\left(Q_{t}, \tilde{\varepsilon_{t}}\right)
$$

onde $Q$ é a quantidade demandada total e $\tilde{\epsilon}_{t}$ é uma variável aleatória com distribuição conhecida. Existem $n$ firmas idênticas que produzem o bem com defasagem de um período, com a seguinte função de produção

$$
\tilde{q}_{t}=f\left(l_{t-1}, \tilde{\theta}_{t}\right)
$$

onde $l_{t-1}$ é a quantidade de insumo utilizada no períỏo $t-1$ para produzir o bem no período $t$ e $\tilde{\theta}_{t}$ é um choque aleatório que afeta a produção do bem no tempo $t$, com distribuição conhecida. Como $Q_{t}=n q_{t}$, aplicando (4) em (3) observamos que $\tilde{p_{t}}=\tilde{p_{t}}\left(\tilde{\varepsilon_{t}}, \tilde{\theta_{t}}\right)$. O preço do insumo no período $t$ é $w_{t}$, não-estocástico. As firmas são competitivas, neutras ao risco. A decisão de produção é feita no período $t-1$, quando as firmas ainda não sabem qual a realização das variáveis aleatórias $\left(\tilde{\varepsilon}_{t}, \tilde{\theta}_{t}\right)$. A função lucro esperado de uma firma é

$$
E \mathrm{II}=E\left[\tilde{p}\left(\tilde{\varepsilon}_{t}, \tilde{\theta}_{t}\right) f\left(l_{t-1}, \tilde{\theta}\right)-w_{t-1} l_{t-1}\right]
$$

Para este modelo, podemos definir EER como

Definição 2. Um EER para o modelo acima é uma quantidade $l_{t-1}^{*}>0$ de insumo para cada firma, e uma distribuição de preços $\tilde{p}^{*}\left(\tilde{\varepsilon}_{t}, \tilde{\theta}_{t}\right)$, tal que

$$
\begin{aligned}
\tilde{p}^{*}\left(\tilde{\varepsilon}_{t}, \tilde{\theta}_{t}\right) & =D\left(n f\left(l_{t-1}^{*}, \tilde{\theta}_{t}\right), \tilde{\varepsilon}_{t}\right) e \\
l_{t-1}^{*} & =\arg _{l_{t-1}} \max E \Pi \\
& =E\left[\tilde{p}^{*}\left(\tilde{\varepsilon}_{t}, \tilde{\theta}_{t}\right) f\left(l_{t-1}, \tilde{\theta}\right)-w_{t-1} l_{t-1}\right] .
\end{aligned}
$$

a expressão (6) mostra que a distribuição das alocações gera a distribuição dos preços. A expressão (7) mostra que a distribuição das alocações é ótima para a distribuição de preços, conforme definição 1.

${ }^{1} \mathrm{O}$ símbolo $\sim$ acima de uma variável $x$ significa que é variável aleatória. Realizaçōes desta variável aleatória serão representados pela variável sem o $\sim$. Assim, se $\bar{x}$ representa a variável aleatória, $x$ representa uma realização desta variável aleatória. 
Esse modelo deixa também bastante claro que o conceito de EER é uma generalização para economias estocásticas do conceito equilíbrio com previsão perfeita de Hicks (1939) para economias nãoestocásticas. Hicks sugeriu o conceito de "equilíbrio com previsão perfeita" para a situação em que o preço que os produtores antecipavam quando tomavam decisões de produção eram os preços que de fato ocorriam como conseqüência das decisões de produção. No EER, a distribuição dos preços antecipada pelos produtores quando fazem suas decisões de produção é a distribuiçâao dos preços que de fato ocorre como conseqüência das decisões de produção.

\section{Expectativas racionais com assimetria de informaçāo: o papel dos preços na alocaçāo dos recursos.}

Na teoria microeconômica padrão, os preços entram no problema do consumidor apenas para definir a restrição orçamentária. Todavia, é uma idéia bastante antiga Hayek (1945) que os preços têm um papel informativo importante. A década de 40 assistiu a um grande debate na literatura de planejamento econômico. Oskar Lange argumentava que um planejador podia gerar a mesma alocação que um sistema de mercado, bastando para isso que o planejador anunciasse o vetor de preços de equilíbrio de mercado como o vetor de preços da economia planejada.

Hayek argumentava que essa linha de raciocínio perdia o aspecto mais importante dos preços: que o sistema de preços numa economia de mercado gera uma fantástica economia de informação. Cada agente sabe de alguma coisa sobre si e sua vizinhança. Transacionando, essas peças de informação dispersas na economia são agregadas e transmitidas pelos preços. Essas idéias foram formalizadas em Lucas (1972) e Green (1973) e numa notável série de artigos de Grossman (1976, 1977, 1978, 1981). O insight fundamental, feito por Lucas é que: se os preços são função da realização de informação e os agentes econômicos sabem a relação entre preços e informação, então quando os agentes observam os preços vigentes, eles podem inferir, pelo menos parcialmente, qual a informação presente no mercado. Nessa seção, apresentaremos um modelo simples ${ }^{2}$ que captura

${ }^{2}$ Desenvolvido em Grossman (1979). 
os aspectos essenciais do insight de Lucas.

Considere a modificação do modelo da seção anterior onde a demanda no período $t$ por um certo bem perecível é estocástica devido a choques aleatórios $\tilde{s}_{t}$. Suponhamos que existam $n$ produtores com mesma tecnologia não-estocástica expressa pela função de custo crescente convexa $C(q)$, onde $q$ é a quantidade produzida. A produção desse bem consome um período de tempo, de forma que a decisão de produção desse bem para o período $t$ é feita no período $t-1$. O produtor $i$ observa, no período $t-1$, antes de tomar sua decisão de produção, a realização da variável aleatória $\bar{y}_{i t-1}$. Seja $\bar{y}_{t-1}=\left(\bar{y}_{1 t-1}, \bar{y}_{2 t-1}, \ldots, \bar{y}_{n t-1}\right)$ o vetor de informação de todos os produtores. É de conhecimento comum ${ }^{3}$ a distribuição conjunta $\left(\tilde{s}_{t}, \tilde{y}_{1 t-1}, \tilde{y}_{2 t-1}, \ldots, \bar{y}_{n t-1}\right)$. Essa distribuição conjunta é i.i.d. no tempo, portanto podemos escrever $\left(\tilde{s}, \bar{y}_{1}, \tilde{y}_{2}, \ldots, \tilde{y}_{n}\right)$. Uma possível definição de equilíbrio para esse modelo é:

Definição 3. Um equilibrio com expectativas realizadas para o período $t-1$ é uma função de preços $\tilde{p}^{0}(\tilde{s}, \tilde{y})$ e uma função de quantidades $q_{i t-1}^{0}\left(\bar{y}_{i}\right)$, tal que

$$
\begin{aligned}
& q_{i t-1}^{0}\left(y_{i}\right)=\arg \max _{q} E\left[\tilde{p}^{0}(\tilde{s}, \tilde{y}) \mid \tilde{y}_{i}=y_{i}\right] q-C(q) ; e \\
& \left.\tilde{p}^{0}(\tilde{s}, \tilde{y})\right)=\cdot D\left(\sum_{i=1}^{n} q_{i t-1}^{0}\left(y_{i}\right), \tilde{s}\right) \forall(\tilde{s}, \tilde{y}) .
\end{aligned}
$$

No período $t-1$, o preço esperado pelo produtor $i$ para o período $t$ é $E\left[\tilde{p}^{0}(\tilde{s}, \tilde{y}) \mid \tilde{y}_{i}=y_{i}\right]$. Como a oferta do produtor $i$ depende de $\tilde{y}_{i}$, o equilíbrio entre oferta e demanda implica que a distribuição de preços de equilíbrio dependa de $\tilde{y}$. Como na definição 1, a expressão (9) mostra que a distribuição das alocações gera a distribuição dos preços, a expressão (8) mostra que a distribuição das alocações é ótima para a distribuição de preços. Entretanto, uma boa definição de EER para economias com informação assimétrica requer algo mais.

$3 \mathrm{Um}$ fato econômico é de conhecimento comum se todos os participantes na economia o conhecem, sabem que os outros conhecem, sabem que os outros sabem que ele conhece, iterando ad infinitum. 
No período $t$, quando os produtores observam o preço de equilíbrio realizado, eles aprendem alguma coisa sobre as informações dos outros produtores. Mas essa informação chega muito tarde. As decisões de produção foram feitas no período $t-1$. Entretanto se $E\left[\tilde{p}^{0}(\tilde{s}, \tilde{y}) \mid \tilde{y}_{i}=y_{i}\right]$ for diferente entre os produtores, existem forças induzindo a que se abra mercado futuro para esse bem no período $t-1$. Se $E\left[\tilde{p}^{0}(\tilde{s}, \tilde{y}) \mid \tilde{y}_{i}=y_{i}\right]>E\left[\tilde{p}^{0}(\tilde{s}, \tilde{y}) \mid \tilde{y}_{j}=y_{j}\right]$ para algum $i$ e $j$, então existe oportunidade de trocas entre esses dois produtores no mercado futuro, pois o produtor $i$ está disposto a pagar um preço maior que o preço esperado pelo produtor $j$. Mas se se abre mercado futuro, o equilíbrio da definição 3 é inadequado para analisar situações com crenças diferentes. Se $p_{f}$ é o preço de equilíbrio no mercado futuro e $E\left[\tilde{p}^{0}(\tilde{s}, \tilde{\mathrm{y}}) \mid \tilde{y}_{i}=y_{i}\right]>E\left[\tilde{p}^{0}(\tilde{s}, \tilde{\mathrm{y}}) \mid \tilde{y}_{j}=y_{j}\right]$ para algum $i$ e $j$, pelo menos uma das duas situações ocorre: $E\left[\tilde{p}^{0}(\tilde{s}, \tilde{\mathrm{y}}) \mid \tilde{y}_{i}=y_{i}\right]>p_{f}$ ou $p_{f}>E\left[\tilde{p}^{0}(\tilde{s}, \tilde{y}) \mid \tilde{y}_{j}=y_{j}\right]$. Como os produtores são neutros ao risco, não pode haver equilíbrio no mercado futuro se os produtores têm crenças diferentes pois demanda e/ou oferta seriam não-limitadas.

Mas com pequenas modificações podemos analisar definições de equilíbrio mais interessantes para esse modelo em mercado futuro. Para simplificar, suporemos que a demanda no mercado spot pelo bem é infinitamente elástica, isto é, não dependa da quantidade transacionada. Assim, a demanda, pode ser expressa como $\tilde{D}=D(\tilde{s})$. O produtor $i$ é agora averso ao risco, com função de utilidade Von Neumman-Morgenstern

$$
u_{i}(w)=1-e^{-a_{i} w}, a_{i}>0
$$

onde $w$ é a riqueza do produtor $i$ e $a_{i}$ é seu coeficiente de aversão ao risco ${ }^{4}$. Suporemos também que $(\tilde{D}, \tilde{y})$, a demanda pelo produto no mercado spot no período $t$ e a informação dos produtores no período $t-1$ tenham distribuição normal conjunta e que apenas os produtores participam do mercado futuro ${ }^{5}$. Seja $x_{i}$ a quantidade vendida do bem pelo produtor $i$ no mercado futuro. A função objetivo do produtor $i$

\footnotetext{
${ }^{4}$ Se $u(w)$ é a função utilidade de um agente, seu coeficiente de aversão ao risco é $-u^{\prime \prime}(w) / u^{\prime}(w)$.

${ }^{5} \mathrm{~A}$ relação intertemporal do modelo é a seguinte. No período $t-1$ ocorrem a realização de informações sobre a demanda spot no período $t$ e as decisões de
} 
é

$$
\max _{q_{i}, x_{i}} E\left[u_{i}\left(p_{f} x_{i}+\left(q_{i}-x_{i}\right) \tilde{D}-C\left(q_{i}\right)\right) \mid y_{i}\right]
$$

Essa função objetivo é equivalente à 6

$$
\begin{aligned}
\max _{q_{i}, x_{i}} E\left[p_{f} x_{i}+\right. & \left.\left(q_{i}-x_{i}\right) \tilde{D}-C\left(q_{i}\right) \mid y_{i}\right] \\
& -\frac{a_{i}}{2} \operatorname{var}\left[p_{f} x_{i}+\left(q_{i}-x_{i}\right) \tilde{D}-C\left(q_{i}\right) \mid y_{i}\right] \\
= & \max _{q_{j}, x_{j}} p_{f} x_{i}+\left(q_{i}-x_{i}\right) E\left[\tilde{D} \mid y_{i}\right] \\
& -C\left(q_{i}\right)-\frac{a_{i}}{2}\left(q_{i}-x_{i}\right)^{2} \operatorname{var}\left[\tilde{D} \mid y_{i}\right]
\end{aligned}
$$

As condições de primeira ordem do problema do produtor são

$$
\begin{aligned}
& p_{f}-E\left[\tilde{D} \mid y_{i}\right]+a_{i}\left(q_{i}-x_{i}\right) \operatorname{var}\left[\tilde{D} \mid y_{i}\right]=0 \\
& E\left[\tilde{D} \mid y_{i}\right]-C^{\prime}\left(q_{i}\right)-a_{i}\left(q_{i}-x_{i}\right) \operatorname{var}\left[\tilde{D} \mid y_{i}\right]=0
\end{aligned}
$$

demandas do produto no mercado futuro no período $t-1$ e de produçōes para atender as demandas do mercado futuro do período $t-1$ e no mercado spot no período $t$. No período $t$ as trocas são realizadas. De fato, essas realizaçōes de informação, decisōes e mercados são totalmente independentes do que ocorrem nos outros períodos, portanto podem ser tratados separadamente. Com isso po. demos eliminar as referências ao tempo nas variáveis. Sempre que nós referirmosà informação, estamos falando da informação que chega no periodo $t-1$ sobre a demanda no mercado spot no período $t$. Sempre que nos referirmos ao mercado futuro, estamos falan do do mercado futuro no período $t-1$ de contratos com maturação no período seguinte. Sempre que nos referirmos às decisōes de produção estamos referindo-nos às decisōes de produção no período $t-1$. Sempre que nos referirmos ao mercado spot estamos falando do mercado spot no período $t$.

${ }^{6}$ Seja $w \sim N\left(\mu, \sigma^{2}\right)$. Pela função geratriz de momentos da normal $M(t)=$ $E\left[e^{t w}\right]=\mathrm{c}^{t e} \exp \left\{t \mu+\frac{t^{2} \sigma^{2}}{2}\right\}$, onde $t$ é a ordem do momento. Então podemos

$$
E\left[u_{i}\right] \approx 1-\exp \left\{-a_{i}\left[\mu-\frac{\alpha_{i} \sigma^{2}}{2}\right]\right\}
$$

Essa função é monótona crescente em $\left[\mu-\frac{\alpha_{i} \sigma^{2}}{2}\right]$ e portanto maximizar $\left(^{*}\right)$ é equivalente a maximizar $\left[\mu-\frac{\alpha_{i} \sigma^{2}}{2}\right]$. 
A solução do sistema (12)-(13) nos dá a demanda e oferta do produtor $i$

$$
\begin{aligned}
x_{i} & =q_{i}+\frac{p_{f}-E\left[\tilde{D} \mid y_{i}\right]}{a_{i} \operatorname{var}\left[\tilde{D} \mid y_{i}\right]}, \\
C^{\prime}\left(q_{i}\right) & =p_{f} .
\end{aligned}
$$

A condição de equilíbrio do mercado futuro é

$$
\sum_{i=1}^{n} x_{i}=0
$$

Para simplificar as expressões suporemos que $C^{\prime}\left(q_{i}\right)=c q_{i}$ (custo marginal linear). Nesse caso obtemos de (15) que $q_{i}=\frac{p_{f}}{c}$. Substituindo essa expressão em (14) e aplicando na condição de equilíbrio do mercado futuro (16) obtemos

$$
p_{f}^{0}(\mathrm{y})=\sum_{i=1}^{n} \frac{\frac{E\left[\tilde{D} \mid y_{i}\right]}{u_{i} \operatorname{var}\left[\tilde{D} \mid y_{i}\right]}}{\sum_{j=1}^{n}\left(a_{j} \operatorname{var}\left[\tilde{D} \mid y_{j}\right]\right)^{-1}+\frac{n}{c}} .
$$

Embora alguns autores sugiram que a expressão (17) (que é um equilíbrio com expectativas realizadas) mostre como preços competitivos agregam informação, na verdade esta equação mostra o contrário. De fato há algo de errado com o equilíbrio (17). Para ver isto, imaginemos que essa economia se replique muitas vezes ao longo do tempo e que o equilíbrio (17) venha sendo obtido em cada vez. O produtor $i$ então observará muitas realizações de $\left(\tilde{p}_{f}^{0}(\tilde{\mathbf{y}}), \tilde{y}_{i}, \tilde{D}\right)$. Com o passar do tempo ele aprenderá a distribuição condicional de $\tilde{D}$ dados $y_{i}$ e $\tilde{p}_{f}^{0}(\mathrm{y})$. Ele geralmente encontrará que $E\left[\tilde{D} \mid y_{i}\right] \neq E\left[\tilde{D} \mid y_{i}, \tilde{p}_{f}^{0}(\mathrm{y})\right] . \tilde{p}_{f}^{0}(\mathrm{y})$ lhe revelará alguma informação adicional à sua informação privada $y_{i}$. Portanto, após observarem o preço de equilíbrio no mercado futuro, os produtores desejarão recontratar. Quando eles recontratarem possivelmente $\tilde{p}_{f}^{0}(\mathrm{y})$ não será mais o preço de equih́brio preço de equilíbrio quando eles maximizavam $E\left[u_{i} \mid y_{i}\right]$. Agora que 
eles maximizam $E\left[u_{i} \mid y_{i}, p_{f}^{0}(\mathbf{y})\right]$ as demandas e ofertas serão diferentes. O conceito de equilíbrio correto para essa situação é o preço $\tilde{p}_{f}^{*}(\mathbf{y})$, tal que se os produtores condicionarem a utilidade esperada em suas informações privadas e no preço de equilíbrio, eles não tenham desejo de recontratar. Este é o EER para esse modelo, que definimos formalmente abaixo.

Definição 4. Um EER para o período $t-1$ é uma função de preços $\tilde{p}_{f}^{*}(\tilde{y})$, funções de quantidades $q_{i}^{*}\left(\tilde{y}_{i}, \tilde{p}_{f}^{*}(\tilde{\mathrm{y}})\right)$ e $x_{i}^{*}\left(\tilde{y}_{i}, \tilde{p}_{f}^{*}(\tilde{\mathrm{y}})\right)$, tal que

$$
\begin{aligned}
& \left(x_{i}^{*}\left(y_{i}, \tilde{p}_{f}^{*}(\mathbf{y})\right) ; q_{i}^{*}\left(\tilde{\gamma} y_{i}, \tilde{p}_{f}^{*}(\mathbf{y})\right)\right) \\
& \quad=\arg \max _{q_{j}, x_{i}} E\left[u_{i}\left(\tilde{p}_{f}^{*}(\mathbf{y}) x_{i}+\left(q_{i}-x_{i}\right) \tilde{D}-C\left(q_{i}\right)\right) \mid \tilde{y}_{i}\right. \\
& \left.\quad=y_{i}, \tilde{p}_{f}^{*}(\mathbf{y})\right]
\end{aligned}
$$

e

$$
\sum_{i=1}^{n} x_{i}^{*}\left(y_{i}, \tilde{p}_{f}^{*}(\mathrm{y})\right)=0 \quad \forall \tilde{y}
$$

Essa noção de equiḱbrio é a correta para esse modelo pois após a obtenção do equilíbrio, os produtores não têm desejo de recontratar, como no equilíbrio da definição 3. Mas o EER é também uma formalização da idéia de Hayeck sobre o papel fundamental dos preços como agregador das informações dispersas no mercado.

\section{Proposição 1.}

$$
p_{f}^{e}(\mathbf{y})=\sum_{i=1}^{n} \frac{\frac{E[\tilde{D} \mid \mathbf{y}]}{a_{t} v a r[\tilde{D} \mid \mathbf{y}]}}{\sum_{j=1}^{n}\left(a_{j} \operatorname{var}[\tilde{D} \mid \mathbf{y}]\right)^{-1}+\frac{n}{c}}
$$

é um equilibrio com expectativas racionais.

Prova: A hipótese de normalidade conjunta de $(\tilde{D}, \tilde{y})$ implica que $\operatorname{var}[\tilde{D} \mid y]=C^{i e}$ (independe de y). Isto implica que $p_{f}^{e}(\mathrm{y})$ é uma função linear estritamente crescente de $E[\tilde{D} \mid \mathbf{y}]$, logo invertível. Isto é, se um produtor observa $p_{f}^{e}(\mathrm{y})$, invertendo a função ele pode saber 
$E[\tilde{D} \mid \mathrm{y}]$. Em linguagem estatística, $p_{f}^{e}(\mathrm{y})$ é uma estatística suficiente de $E[\tilde{D} \mid \mathbf{y}]$. Isto implica que a expressão (11)

$$
\max _{q_{i}, x_{i}} p_{f} x_{i}+\left(q_{i}^{A}-x_{i}\right) E\left[\tilde{D} \mid y_{i}\right]-C\left(q_{i}\right)-\frac{a_{i}}{2}\left(q_{i}-x_{i}\right)^{2} \operatorname{var}\left[\tilde{D} \mid y_{i}\right]
$$

pode ser escrita' para este caso como

$$
\max _{q_{i}, x_{i}} p_{f} x_{i}+\left(q_{i}-x_{i}\right) E[\tilde{D} \mid \mathbf{y}]-C\left(q_{i}\right)-\frac{a_{i}}{2}\left(q_{i}-x_{i}\right)^{2} \operatorname{var}[\tilde{D} \mid \mathbf{y}]
$$

A solução do problema acima nos dá

$$
\begin{aligned}
& x_{i}^{e}(y)=q_{i}+\frac{p_{f}-E[\tilde{D} \mid \mathbf{y}]}{a_{i} \operatorname{var}[\tilde{D} \mid \mathbf{y}]} \\
& C^{\prime}\left(q_{i}\right)=p_{f}^{e} \\
& \sum_{i=1}^{n} x_{i}^{*}(\mathrm{y})=0 .
\end{aligned}
$$

É fácil verificar que a solução do sistema (21)-(23) nos dá (20).

O fato notável desse equilíbrio com expectativas racionais é que ele é idêntico ao equilíbrio que ocorreria na economia se os produtores possuíssem toda a informação do mercado. O preço do mercado futuro no equilíbrio com expectativas racionais revela toda a informação dispersa no mercado. Na seção seguinte, construiremos um modelo de equilíbrio geral com incerteza e informação assimétrica para estudarmos a existência e propriedades de bem-estar do equilíbrio com expectativas racionais.

\section{Equilibrio geral e propriedades de bem-estar.}

Consideremos uma economia de pura troca com dois períodos, período 1 e período 2 com incerteza apenas no período 2, e mercados futuros (contigentes no estado da natureza) completos. As dotações dos consumidores no período 2 dependem de um estado da natureza aleatório $\tilde{s}$ que pode assumir um número finito $S$ de valores positivos. 
Existe apenas um bem em cada estado da natureza ${ }^{7}$. No período 1 não existe incerteza. Existe um número finito $n$ de consumidores. Seja $w_{i}$ o vetor de dotações do consumidor $i$ onde $\omega_{i 1}$ é a dotação inicial do consumidor $i$ do bem no período 1 e $\omega_{i 2 s}$ é a dotação inicial do consumidor $i$ do bem no período 2 no estado da natureza $s$. Vamos representar o consumo do bem pelo consumidor $i$ no período 1 por $c_{i 1}$ e o consumo do bem $s$ pelo consumidor $i$ no período 2 por $c_{i 2 s}$. Sua cesta de consumo planejada é $\mathbf{c}_{i}=\left(c_{i 1}, c_{i 21}, c_{i 22}, \ldots, c_{i 2 s}\right)$. É útil também introduzir as notações $w_{i 2}$ e $c_{i 2}$ para representar as dotações iniciais e os consumos do consumidor $i$ no período 2 . Consumidores têm preferências por consumo representados por uma função de utilidade esperada.

$$
U_{i}\left(\mathbf{c}_{i}\right)=\sum_{s=1}^{s} u_{i}\left(c_{i 1}, c_{i 2 s}\right) \pi_{i s},
$$

onde $\pi_{i s}=\operatorname{Pr}(\bar{s}=s)$ é a probabilidade que o consumidor $i$ associa à ocorrência do estado da natureza $s$ no segundo período. Tomamos o bem no período 1 como numerário e representamos o preço do bem no período 2 no estado da natureza $s$ por $p_{s}$. O vetor de preços é representado por $\mathbf{p}=\left(1, p_{1}, p_{2}, \ldots, p_{s}\right)$. $\mathbf{p}_{2}$ representará o vetor de preços do bem no período 2 nos diversos estados da natureza. A assimetria de informação no modelo entra da seguinte forma. No período 1 , o consumidor $i$ obtém informações sobre a distribuição de probabilidades de $\tilde{s}$ no período 2. Essa informação é a observação no período 1 de uma variável aleatória $\tilde{y}_{i}$ com contra-domínio $Y_{i}$. O vetor da informação presente em toda a economia é representado por $\tilde{\mathbf{y}}=\left(\tilde{y}_{1}, \tilde{y}_{2}, \ldots, \tilde{y}_{n}\right)$, com contra-domínio $\mathrm{Y} \subset R^{n}$. $\tilde{\mathbf{y}}$ tem uma distribuição conjunta com $\tilde{s}$ conhecida por todos $(\tilde{s}, \tilde{y}) \cdot{ }^{8}$ Essas informações chegam aos consumidores antes dos mercados abrirem. Para facilitar a visualização da seqüência dos acontecimentos no tempo, vamos representá-la no gráfico abaixo.

\footnotetext{
${ }^{7} \mathrm{~A}$ hipótese de existir apenas um bem para cada estado da natureza é feita apenas para simplificar notação. É fácil verificar que todos os resultados permanecem válidos se existirem muitos bens por estados da natureza.

${ }^{8}$ Em linguagem mais precisa, essa distribuição conjunta é de conhecimento comum. Como todos os consumidores possuem a mesma função de probabilidade, podemos suprimir o índice $i$ na definição desta função.
} 


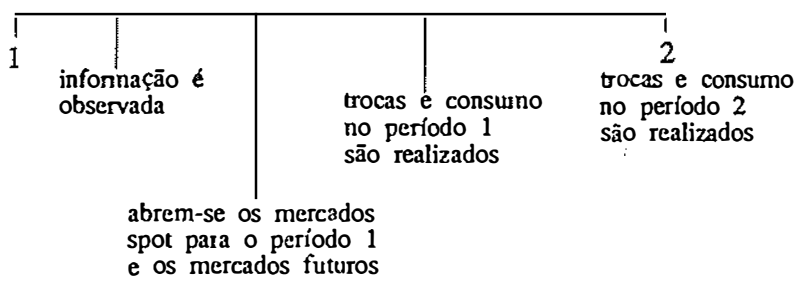

Recordando que fizemos a suposição de existência de um conjunto completo de mercadorias contingentes de Arrow-Debreu, isto é, os consumidores podem vender e comprar promessas de entrega e recebimentos de mercadorias contigentes à ocorrência do estado $s$ no período 2, podemos definir equilíbrio Walrasiano.

Definição 5. Um equilíbrio de Arrow-Debreu (ou Walrasiano) para essa economia é, para cada realização possivel de $\mathrm{y} \in \mathrm{Y}$, um vetor de preços $\mathrm{p}^{\mathrm{W}}(\mathrm{y})$ e vetores de alocações $\mathbf{c}_{i}^{\mathrm{W}}(\mathrm{y})$, um para cada $i$, tal $q u e^{9}$

$$
\begin{aligned}
& c_{i}^{\mathrm{W}}\left(y_{i}\right)=\arg \max _{c_{i}} \sum_{s=1}^{S} u_{i}\left(c_{i 1}, c_{i 2 s}\right) \pi_{s}\left(y_{i}\right) \\
& \text { s.a. } \\
& c_{i 1}^{\mathrm{W}}\left(y_{i}\right)+\mathbf{p}^{\mathrm{W}}(\mathrm{y}) \cdot c_{i 2}^{\mathrm{W}}\left(y_{i}\right) \leq \mathrm{W}_{i l}+\mathbf{p}^{\mathrm{W}}(\mathrm{y}) \cdot \mathrm{W}_{i 2}, \forall i ; \quad \mathrm{e} \\
& \sum_{i=1}^{n} \mathrm{c}_{i}^{\mathrm{W}}\left(\mathrm{y}_{i}\right) \leq \sum_{i=1}^{n} \mathrm{~W}_{i},
\end{aligned}
$$

onde $\pi_{s}\left(\mathrm{y}_{i}\right)=\operatorname{Pr}\left(\tilde{s}=s \mid \tilde{\mathrm{y}}_{i}=\mathrm{y}_{i}\right)$. Designemos um equilíbrio Walrasiano dessa economia por $\tilde{\mathbf{p}}^{\mathrm{W}}(\tilde{\mathbf{y}}), \tilde{\mathbf{c}}_{i}^{\mathrm{W}}(\tilde{\mathbf{y}})$. Observe que as crenças dos consumidores $i$ (a respeito de $\tilde{s}$ ) dependem da informação privada $\tilde{\mathbf{y}}_{i}$. Portanto o vetor preços que faz com que não exista excesso de demanda depende de toda a informação da economia. Como paradigma

${ }^{9} \mathrm{O}$ símbolo ". " representa "produto interno". 
para se comparar diferentes tipos de equilíbrio desse modelo, vamos definir o equilíbrio Walrasiano para uma economia artificial onde cada consumidor possui toda a informação da economia: cada consumidor observa no período 1 todo o vetor $\tilde{y}$. Nessa economia artificial, cada consumidor maximiza $E\left[u_{i} \mid \tilde{y}\right]=\sum_{s=1}^{s} u_{i}\left(c_{i 1}, \mathbf{c}_{i 2 s}\right) \pi_{s}(\tilde{\mathbf{y}})$. Seja $\tilde{\mathbf{p}}^{a}(\tilde{\mathbf{y}})$ e $\tilde{\mathbf{c}}_{i}^{a}(\tilde{\mathbf{y}})$ um equilíbrio Walrasiano para essa economia artificial, isto é, para cada y $\in \mathbf{Y}, \mathbf{p}^{a}(\mathbf{y})$ e $\mathbf{c}_{i}^{a}(\mathbf{y})$ satisfazem (25) e (26) quando $\pi_{s}\left(\mathrm{y}_{i}\right)$ é substituído por $\pi_{s}(\mathrm{y})$. Portanto, pelo primeiro teorema fundamental da teoria do bem-estar ${ }^{10}$, um planejador central com toda informação da economia não poderia encontrar uma alocação factível $\left\{\tilde{\mathbf{c}}_{i}(\tilde{\mathbf{y}})\right\}_{i=1}^{n}$, tal que

$$
\begin{array}{lll}
E\left[u_{i}\left(c_{i 1}, c_{i 2 \bar{s}}\right) \mid \mathrm{y}\right] \geq E\left[u_{i}\left(c_{i 1}^{a}, c_{i 2 \bar{s}}^{a}\right) \mid \mathrm{y}\right] & \forall \mathrm{y}, \forall i \text { e } \\
E\left[u_{i}\left(c_{i 1}, c_{i 2 \bar{s}}\right) \mid \mathrm{y}\right]>E\left[u_{i}\left(c_{i 1}^{a}, c_{i 2 \bar{s}}^{a}\right) \mid \mathrm{y}\right] & \text { para algum } \mathrm{y}, i
\end{array}
$$

No entanto, o equilíbrio Walrasiano para a economia com assimetria de informação pode em geral ser Pareto dominado por um planejador central com toda informação. Pode existir $\left\{\tilde{\mathbf{c}}_{i}(\tilde{\mathbf{y}}\}_{i=1}^{n}\right.$ factível tal que

$$
\begin{array}{lll}
E\left[u_{i}\left(c_{i 1}, c_{i 2 \tilde{s}}\right) \mid \mathrm{y}\right] \geq E\left[u_{i}\left(c_{i 1}^{\mathrm{W}}, c_{i 2 \tilde{s}}^{\mathrm{W}}\right) \mid \mathrm{y}\right] & \forall \mathbf{y}, \forall \mathbf{i} \text { e } \\
E\left[u_{i}\left(c_{i 1}, c_{i 2 \bar{s}}\right) \mid \mathrm{y}\right]>E\left[u_{i}\left(c_{i 1}^{\mathrm{W}}, c_{i 2 \tilde{s}}^{\mathrm{W}}\right) \mid \mathrm{y}\right] & \text { para algum } \mathbf{y}, \mathbf{i} .
\end{array}
$$

Definição 6. Um EER para essa economia é, para cada realização possivel de $y \in \mathrm{Y}$, um vetor de preços $\mathbf{p}^{*}(\mathbf{y})$ e vetores de alocações $\mathbf{c}_{i}^{*}\left(\mathbf{y}, \mathbf{p}^{*},(\mathbf{y})\right)$, um para cada $i$, tal que

$$
\begin{aligned}
& \mathbf{c}_{i}^{*}\left(y_{i}, \mathbf{p}^{*}(\mathbf{y})\right)=\arg \max _{c_{1}} \sum_{s=1}^{s} \mathbf{u}_{i}\left(c_{i l}, \mathbf{c}_{i 2 s}\right) \boldsymbol{\gamma}_{s}\left(y_{i}, \mathbf{p}^{*}(\mathbf{y})\right) \\
& \text { s.a. } \\
& c_{i 1}^{*}\left(y_{i}, \mathbf{p}^{*}(\mathbf{y})\right)+\mathbf{p}^{*}(\mathbf{y}) \cdot \mathbf{c}_{i 2}^{*}\left(y_{i}, \mathbf{p}^{*}(\mathbf{y})\right) \leq \omega_{i 1} \\
& +\mathrm{p}^{*}(\mathrm{y}) \cdot \mathrm{w}_{i 2}, \forall i ; \quad e \\
& \sum_{i=1}^{n} \mathrm{c}_{i}^{*}\left(y_{i}, \mathbf{p}^{*}(\mathrm{y})\right) \leq \sum_{i=1}^{n} \mathrm{w}_{i} \text {. }
\end{aligned}
$$

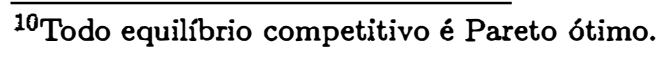


Seguindo a mesma linha de argumentação da seção anterior, vamos provar agora que um equilíbrio da economia artificial é um EER e, conseqüentemente, o EER é Pareto ótimo. Além disso, o EER tem a propriedade de agregar perfeitamente a informação presente na economia em tempo hábil para ser utilizada nas decisões econômicas dos consumidores. Observemos que

$$
\operatorname{Pr}(\tilde{s} \mid \mathrm{y})=\operatorname{Pr}(\tilde{s} \mid \pi(\mathrm{y})) .
$$

Assim, $E\left[u_{i} \mid \mathrm{y}\right]=E\left[u_{i} \mid \pi(\mathrm{y})\right]$ e podemos escrever o equilíbrio da economia artificial como $\left(\tilde{p}^{a}(\pi(\tilde{y})), \tilde{c}^{a}(\pi(\tilde{y}))\right)$. Existem $S+1$ mercados e $S$ estados da natureza. Assim, o vetor de preços de equilíbrio da economia artificial $\tilde{p}^{a}(\pi(\tilde{y}))$ é uma função de $R^{s-1}$ em $R^{s}$. Suponhamos que ela seja invertível. Neste caso, observar $\tilde{\mathbf{p}}^{a}(\pi(\tilde{\mathbf{y}}))$ é equivalente a observar $\pi(\tilde{y})$. Isto implica que o equilibrio da economia artificial é um EER, porque neste caso maximizar $E\left[u_{i} \mid y_{i}, p^{a}(\mathrm{y})\right]=$ $E\left[u_{i} \mid y_{i}, \pi(\mathrm{y})\right]=E\left[u_{i} \mid \pi(\mathrm{y})\right]$ (esta última igualdade é dada por (29)). A proposição abaixo mostra que $\tilde{\mathrm{p}}^{a}(\pi(\tilde{\mathrm{y}}))$ é invertível.

Proposição 2. Suponhamos que todos os consumidores são localmente nâo-saciáveis, para todo $i, s, u_{i}\left(c_{i 1}, c_{i 2 s}\right)$ é uma função diferenciável estritamente côncava e aditivamente separável no tempo $\left(u_{i}\left(c_{i l}, c_{i 2 s}\right)=u_{i 1}\left(c_{i 1}\right)+u_{i 2}\left(c_{i 2 s}\right)\right)$, para todo $\mathrm{y}, i \pi_{s}(\mathrm{y})>0$. Sejam y e $\overline{\mathbf{y}}$ duas realizações distintas de $\overline{\mathbf{y}}$ que gerem $\pi(\mathrm{y})=\pi$ e $\pi(\overline{\mathbf{y}})=\bar{\pi}$ tal que $\pi \neq \bar{\pi}$. Se existir consumidor $j$ tal que $\mathbf{c}_{j}(\mathbf{y}) \neq \mathbf{c}_{j}(\overline{\mathbf{y}})$, então $\mathbf{p}^{a}(\mathbf{y}) \neq \mathbf{p}^{a}(\overline{\mathbf{y}})^{11}$.

Prova: (Por contradição) Suponhamos que $\mathbf{p}^{a}(\mathbf{y})=\mathbf{p}^{a}(\overline{\mathrm{y}})=\mathbf{p}$ e que exista um consumidor $j$ tal que $\mathbf{c}_{j}^{a}(\mathbf{y}) \neq \mathbf{c}_{j}^{a}(\overline{\mathbf{y}})$. Sejam $\mathrm{c}_{j}^{a}(\mathrm{y})=\left(c_{j 1}, c_{j 21}, c_{j 22}, \ldots, c_{j 2 s}\right)$ e $c_{j}^{a}(\overline{\mathrm{y}})=\left(\bar{c}_{j 1}, \bar{c}_{j 21}, \bar{c}_{j 22}, \ldots, c_{j 2 s}\right)$. Como ambas as cestas são compráveis, por preferência revelada

$$
\sum_{s} u_{j}\left(c_{j 1}, c_{j 2 s}\right) \pi_{s}>\sum_{s} u_{j}\left(\bar{c}_{j 1}, \bar{c}_{j 2 s}\right) \pi_{s}
$$

${ }^{11} \mathrm{O}$ que estamos provando é que $p^{a}(\pi(y))$ é essencialmente invertivel. Se para $\mathbf{y}, \overline{\mathbf{y}} \in \mathbf{Y}, \pi(\mathbf{y}) \neq \pi(\overline{\mathbf{y}})$ mas $\left\{c_{i}^{a}(\pi(y))\right\}_{i=1}^{n}=\left\{c_{i}^{a}(\pi(\bar{y}))\right\}_{i=1}^{n}$, não é preciso que $p^{a}(\pi(y)) \neq p^{a}(\pi(\overline{(y)}))$. Ou seja, se quando informação diferente $y, \overline{\mathbf{y}} \in \mathbf{Y}$ leva à mesma demanda de equilíbrio, não é necessário que os preços de equilíbrio sejam distintos. 
Por concavidade estrita de $u_{j}(\cdot, \cdot)$

$$
\begin{aligned}
u_{j}\left(c_{j 1}, c_{j 2 s}\right)-u_{j}\left(\bar{c}_{j 1}, \bar{c}_{j 2 s}\right) & <\frac{\partial u_{j}\left(\bar{c}_{j 1}, \bar{c}_{j 2 s}\right)}{\partial c_{1}}\left(c_{j 1}-\bar{c}_{j 1}\right) \\
& +\frac{\partial u_{j}\left(\bar{c}_{j 1}, \bar{c}_{j 2 s}\right)}{\partial c_{2 s}}\left(c_{j 2 s}-\bar{c}_{j 2 s}\right) .
\end{aligned}
$$

Combinando (30) e (31), obtemos

$$
\sum_{s}\left[\frac{\partial u_{j}\left(\bar{c}_{j 1}, \bar{c}_{j 2 s}\right)}{\partial c_{1}}\left(c_{j 1}-\bar{c}_{j 1}\right)+\frac{\partial u_{j}\left(\bar{c}_{j 1}, \bar{c}_{j 2 s}\right)}{\partial c_{2 s}}\left(c_{j 2 s}-\bar{c}_{j 2 s}\right)\right] \pi_{s}>0 .
$$

Das condições de $1 \underline{a}$ ordem do problema do consumidor $j, \exists \bar{\lambda}>0$ tal que

$$
\begin{gathered}
\sum_{s}\left[\frac{\partial u_{j}\left(\bar{c}_{j 1}, \bar{c}_{j 2 s}\right)}{\partial c_{1}}\right] \bar{\pi}_{s} \leq \bar{\lambda}_{j},=\text { if } \quad \bar{c}_{j 1}>0 \\
\frac{\partial u_{j}\left(\bar{c}_{j 1}, \bar{c}_{j 2 s}\right)}{\partial c_{2 s}} \bar{\pi}_{s} \leq \bar{\lambda}_{j} p_{s}=i f \quad \bar{c}_{j 2 s}>0 .
\end{gathered}
$$

Combinando (32) e (33) obtemos

$$
\sum_{s} \frac{\partial u_{j}\left(\bar{c}_{j 1}, \bar{c}_{j 2 s}\right)}{\partial c_{1}}\left(c_{j 1}-\bar{c}_{j 1}\right) \pi_{s}+\bar{\lambda}_{j} \sum_{s} p_{s}\left(c_{j 2 s}-\bar{c}_{j 2 s}\right) \frac{\pi_{s}}{\bar{\pi}_{s}}>0
$$

onde fizemos uso do fato que se $\bar{c}_{j 2 s}=0$, sabemos que $\left(c_{j 2 s}-\bar{c}_{j 2 s}\right) \geq 0$. Pela hipótese de função de utilidade aditivamente separável no tempo, temos que

$$
\sum_{s} \frac{\partial u_{j}\left(\bar{c}_{j 1}, \bar{c}_{j 2 s}\right)}{\partial c_{1}} \pi_{s}=\sum_{s} \frac{\partial u_{j}\left(\bar{c}_{j 1}, \bar{c}_{j 2 s}\right)}{\partial c_{1}} \bar{\pi}_{s}
$$

Fazendo novamente uso do fato que se $\bar{c}_{j 1}=0$, sabemos que $\left(c_{j 1}-\right.$ $\left.\bar{c}_{j 1}\right) \geq 0$ e de que $\bar{\lambda}_{j}>0$, aplicando (35) em (34), combinando com (33) e dividindo ambos os lados de (34) por $\bar{\lambda}_{j}>0$, obtemos

$$
\sum_{s}\left[\left(c_{j 1}-\bar{c}_{j 1}\right)+p_{s}\left(c_{j 2 s}-\bar{c}_{j 2 s}\right)\right] \frac{\pi_{s}}{\bar{\pi}_{s}}>0 .
$$


A desigualdade (36) vale para todo consumidor $i$ tal que $\mathbf{c}_{i} \neq \overline{\mathbf{c}}_{i} \mathrm{e}$ vale como igualdade para todo $i$ tal que $\mathbf{c}_{i}=\overline{\mathbf{c}}_{i}$. Somando (36) sobre $i$, obtemos que

$$
\sum_{s} \sum_{i}\left(c_{i 1}-\bar{c}_{i 1}\right)+\sum_{s} p_{s} \frac{\pi_{s}}{\bar{\pi}_{s}} \sum_{i}\left(c_{i 2 s}-\bar{c}_{i 2 s}\right)>0 .
$$

Mas (37) contradiz o fato que por não-saciedade local $\sum_{i} c_{i 1}=\sum_{i} \bar{c}_{i 1}$ e $\sum_{i} c_{i 2 s}=\sum_{i} \bar{c}_{i 2 s}$.

Teorema 1. Nas hipóteses da proposição 2, se existe um equilíbrio Walrasiano $\left(\tilde{\mathbf{p}}^{\alpha}(\pi(\tilde{\mathbf{y}})), \tilde{\mathbf{c}}^{a}(\pi(\tilde{\mathbf{y}}))\right)$ para a economia artificial perfeitamente informada, então $\left(\tilde{\mathbf{p}}^{a}(\pi(\tilde{\mathbf{y}})), \tilde{\mathbf{c}}^{\alpha}(\pi(\tilde{\mathbf{y}}))\right)$ é um EER para a economia onde cada consumidor $i$ observa $\tilde{y}_{i}$.

Prova: Se $\tilde{\mathbf{p}}^{a}(\pi(\tilde{\mathbf{y}}))$ é invertível, é então idêntico para um consumidor $i$ maximizar $E\left[u_{i} \mid y_{i}, \tilde{p}^{a}(\pi(\tilde{\mathbf{y}}))\right]$ ou $E\left[u_{i} \mid \pi(\tilde{\mathbf{y}})\right]$, logo em ambos os casos terão a mesma demanda. Portanto, $\left(\tilde{\mathbf{p}}^{a}(\pi(\tilde{\mathbf{y}})), \tilde{c}^{a}(\pi(\tilde{\mathbf{y}}))\right)$ é EER.

Suponhamos que $\tilde{\mathbf{p}}^{a}(\pi(\tilde{\mathbf{y}}))$ não é invertível, ou seja, maximizar $E\left[u_{i} \mid y_{i}, p^{a}(\pi(\tilde{y}))\right]$ é diferente de maximizar $E\left[u_{i} \mid \pi(\tilde{y})\right]$ para algum consumidor $i$ e informação y. Sejam y e $\overline{\mathbf{y}}$ duas realizações distintas de $\tilde{\mathbf{y}}$ onde $\tilde{\mathbf{p}}^{a}(\pi(\tilde{\mathbf{y}}))$ não é invertível. Isto é, que gerem $\pi(\mathrm{y})=\pi$ e $\pi(\bar{y})=\bar{\pi}$ tal que $\pi \neq \bar{\pi}$ e que $\mathbf{p}^{a}(\pi(\mathrm{y}))=\mathrm{p}^{a}(\pi(\overline{\mathrm{y}}))=\mathrm{p}$. Pela proposição $1 \mathbf{c}_{j}^{a}(\mathbf{y})=\mathbf{c}_{j}^{a}(\overline{\mathrm{y}})$. Portanto nơs pontos onde $\tilde{\mathbf{p}}^{a}(\pi(\tilde{\mathbf{y}}))$ não é invertível, não importa ao consumidor saber distinguir entre as possíveis realizações de $\pi(\tilde{y})$, o que contradiz a suposição de que maximizar $E\left[u_{i} \mid y_{i}, p^{a}(\pi(\tilde{y}))\right]$ é diferente de maximizar $E\left[u_{i} \mid \pi(\tilde{y})\right]$ para algum consumidor $i$ e informação y.

Como o equilíbrio Walrasiano da economia artificial não pode ser Pareto dominado por um planejador central que saiba de toda a informação presente no mercado, existe um EER que é Pareto ótimo. Formalmente,

Teorema 2. Existe um EER $\left(\tilde{\mathbf{p}}^{*}(\tilde{\mathbf{y}}), \tilde{\mathbf{c}}_{i}^{*}\left(\tilde{\mathbf{y}}, \tilde{\mathbf{p}}^{*}(\tilde{\mathbf{y}})\right)\right)$ tal que se $\tilde{\mathbf{c}}_{i}(\tilde{\mathrm{y}}, \tilde{\mathbf{p}}(\tilde{\mathrm{y}}))$ é qualquer outra alocação factível (isto é, para todo $\left.\mathrm{y}, \sum_{i} \mathbf{c}_{i}(\mathrm{y}) \leq \sum_{i} \mathbf{w}_{i}\right)$, então não pode ser que

$$
\begin{gathered}
E\left[\mathbf{u}_{i}\left(c_{i 1}, c_{i 2 \tilde{s}}\right) \mid \mathbf{y}\right] \geq E\left[u_{i}\left(c_{i 1}^{*}, c_{12 \tilde{s}}^{*}\right) \mid \mathbf{y}_{i}\right] \quad \forall \mathbf{y}, \forall \mathbf{i} e \\
E\left[\mathbf{u}_{i}\left(c_{i 1}, c_{i 2 \tilde{s}}\right) \mid \mathbf{y}\right]>E\left[\mathbf{u}_{i}\left(c_{i 1}^{*}, c_{i 2 \bar{s}}^{*}\right) \mid \mathbf{y}_{i}\right] \text { para algum } \mathbf{y}, i
\end{gathered}
$$


Scria muito bom se todos os EER's revclassem toda a informaçĩo da economia, pelo menos no caso como esse com mercados completos. lnfelizmente isto não é verdade como o exemplo da próxima seção ilustra.

\section{Existência de equilíbrio com expectativas racionais näo- ótinos.}

Vamos apresentar nesta seção dois resultiados: (i) nas condições do modclo da seção 4 existe um EER que não agrega a informaçĩo presente no mercado; (ii) nas mesmas condições, existe um EER que ć Pareto dominado.

Para mostrar o primciro "mau" excmplo, façamos a seguinte simplificação do modelo da seção 4 . Existem apenas dois estados da natureza do período 2, denotados por $s_{1}$ e $s_{2}$, e dois consumidores denotados por 1 e 2 . O consumidor $i$ obscrva a realização de uma variável alcatória $\bar{y}_{i}$, que também podem assumir apenas dois valores distintos: 1 c 2. A distribuição conjunta do estado da natureza e intormaçũess é

$$
\begin{aligned}
& \operatorname{Pr}\left(s_{1}, i, i\right)=\frac{1}{4} a, \text { para } i=1,2 ; \\
& \operatorname{Pr}\left(s_{1}, i, j\right)=\frac{1}{4}(1-a), \text { para } i \neq j ; \\
& \operatorname{Pr}\left(s_{2}, i, i\right)=\frac{1}{4}(1-a), \text { para } i=1,2 ; \\
& \operatorname{Pr}\left(s_{2}, i, j\right)=\frac{1}{4} a, \text { para } i \neq j .
\end{aligned}
$$

Não existe risco social, isto é, a dotação agregada no período é constante cm todos os cstados da natureza que é igual à dotação agregada no período 1 . Scja $\mathbf{w}_{1}=(1 / 2 \omega, \omega, 0)$ a dotação inicial do indivíduo $1 \mathrm{e} \mathrm{w}_{2}=(1 / 2 \omega, 0, \omega)$. Assim, $\omega_{11}+\omega_{21}=\omega_{121}+\omega_{221}=$ $\omega_{122}+\omega_{222}=\omega$.

Definição 7. Um EER para essa economia são três vetores: 


$$
\begin{aligned}
\mathbf{p}^{*}(\mathbf{y}) & =\left(1, p_{1}^{*}(\mathbf{y}), p_{2}^{*}(\mathbf{y})\right) \\
\mathbf{c}_{1}^{*}\left(y_{1}, \mathbf{p}^{*}(\mathbf{y})\right) & =\left(\mathbf{c}_{11}^{*}\left(y_{1}, \mathbf{p}^{*}(\mathbf{y})\right), \mathbf{c}_{121}^{*}\left(y_{1}, \mathbf{p}^{*}(\mathbf{y})\right), \mathbf{c}_{122}^{*}\left(y_{1}, \mathbf{p}^{*}(\mathbf{y})\right)\right) \text { e } \\
\mathbf{c}_{2}^{*}\left(y_{2}, \mathbf{p}^{*}(\mathbf{y})\right) & =\left(\mathbf{c}_{21}^{*}\left(y_{2}, \mathbf{p}^{*}(\mathbf{y})\right), \mathbf{c}_{221}^{*}\left(y_{2}, \mathbf{p}^{*}(\mathbf{y})\right), \mathbf{c}_{222}^{*}\left(y_{2}, \mathbf{p}^{*}(\mathbf{y})\right)\right)
\end{aligned}
$$

tal que

$$
\begin{aligned}
\mathbf{c}_{i}^{*}\left(y_{i}, \mathbf{p}^{*}(\mathbf{y})\right)= & \arg \max _{c_{i 1}, c_{i 21}, c_{i 22}} \mathbf{u}_{1}\left(c_{i 1}\right)+\mathbf{u}_{i}\left(c_{i 21}\right) \operatorname{Pr}\left(s_{1} \mid y_{i}, \mathbf{p}^{*}(\mathbf{y})\right) \\
& +\mathbf{u}_{i}\left(c_{i 22}\right) \operatorname{Pr}\left(s_{2} \mid y_{i}, \mathbf{p}^{*}(\mathbf{y})\right)
\end{aligned}
$$

s.a.

$c_{i 1}+p_{1}^{*} c_{i 21}+p_{2}^{*} c_{i 22}=\omega_{i 1}+p_{1}^{*} \omega_{i 21}+p_{2}^{*} \omega_{i 22}, \quad i=1,2$

e

$$
\left\{\begin{array}{l}
c_{11}^{*}+c_{21}^{*}=\omega \\
c_{11 j}^{*}+c_{21 j}^{*}=\omega, j=1,2
\end{array}\right.
$$

Considere o vetor $\mathbf{p}^{*}=(1,1 / 2,1 / 2)$ para todo $\mathbf{y}$. Neste caso, o consumidor $i$ encontra que $\operatorname{Pr}\left(s_{j} \mid y_{i}, \mathbf{p}^{*}=(1,1 / 2,1 / 2)\right)=\frac{1}{2}$, para $j=$ 1,2 . É fácil ver que $\mathbf{p}^{*}(1,1 / 2,1 / 2), \mathbf{c}_{i}^{*}=(1 / 2 \omega, 1 / 2 \omega, 1 / 2 \omega), i=1,2$ é um EER. Este EER não agrega informação pois o vetor de prȩ̣os é constante em y e portanto não revela qualquer informação. Outra maneira de ver isto é observar que $\operatorname{Pr}\left(s_{j} \mid y_{i}, \mathbf{p}^{*}(\mathbf{y})\right)=\operatorname{Pr}\left(s_{j} \mid y_{i}\right) \neq$ $\operatorname{Pr}\left(s_{j} \mid \mathbf{y}\right)$.

Para mostrar um EER que pode ser dominado no sentido de Pareto, consideremos uma economia com uma estrutura idêntica à descrita acima, exceto por uma amplificação: agora existe produção. Especificamente, cada consumidor escolhe como sua dotação inicial um vetor na combinação convexa de $(1 / 2 \omega, \omega, 0)$ e $(1 / 2 \omega, 0, \omega)$. Consumidores escolhem sua dotação inicial após o fechamento do mercado futuro mas antes da resolução da incerteza.

Nesse caso, para todo $\mathbf{y}, \mathbf{p}^{*}(\mathbf{y})=(1,1 / 2,1 / 2), \mathbf{c}_{i}^{*}(\mathbf{y})=(1 / 2 \omega$, $1 / 2 \omega, 1 / 2 \omega), i=1,2, \mathrm{w}_{i}^{*}(\mathrm{y})=(1 / 2 \omega, 1 / 2 \omega, 1 / 2 \omega), i=1,2$ continua sendo um EER. Todavia um planejador central com toda a informação da economia escolheria $\mathbf{w}_{i}(\mathbf{y})=\left(\omega, \omega_{1}(\mathbf{y}), \omega-\omega_{1}(\mathbf{y})\right)$ e 
$\left.\mathbf{c}_{i}(\mathbf{y})=\mathrm{w}_{i}(\mathrm{y})\right)$ tal que

$$
\begin{aligned}
& \frac{\mathbf{u}_{i}^{\prime}\left(\omega_{1}(\mathrm{y})\right)}{\mathbf{u}_{i}^{\prime}\left(\omega-\omega_{1}(\mathrm{y})\right)}=\frac{a}{1-a}, \text { se } y_{1}=y_{2}, \text { e } \\
& \frac{\mathbf{u}_{i}^{\prime}\left(\omega_{1}(\mathrm{y})\right)}{\mathbf{u}_{i}^{\prime}\left(\omega-\omega_{1}(\mathrm{y})\right)}=\frac{1-a}{a}, \text { se } y_{1} \neq y_{2},
\end{aligned}
$$

que domina $\mathbf{c}_{i}^{*}=(1 / 2 \omega, 1 / 2 \omega, 1 / 2 \omega), i=1,2, \mathrm{w}_{i}^{*}=(1 / 2 \omega, 1 / 2 \omega, 1 / 2 \omega)$, $i=1,2$ no sentido de Pareto.

\section{Expectativas racionais em modelos macroeconômicos ${ }^{12}$.}

Existem dois tipos de modelos macroeconômicos com expectativas racionais: modelos com agentes maximizadores (com fundamentos microeconômicos) e modelos macroeconômicos simples, geralmente log-lineares, de relações entre variáveis macroeconômicas (renda agregada, nível de preços, oferta de moeda etc.). Nos modelos macroeconômicos com fundamentos micro, o conceito de EER é idêntico ao desenvolvido neste artigo. Vamos exemplificar aqui o conceito de EER em modelos log-lineares com um modelo simples. Seja $y$ uma variável macro que segue a seguinte equação de expectativas

$$
y_{t}=a E\left[y_{t+1} \mid \mathrm{t}\right]+c x_{t}
$$

onde $E\left[y_{t+1} \mid \mathrm{t}\right]$ é a esperança condicional de $y_{t+1}$, mantida no período $t$ de forma que $y$ depende da expectativa corrente sobre o seu valor no futuro e de uma outra variável $x$.

Neste modelo definimos expectativas racionais como a esperança matemática de $y_{t+1}$ condicioual na informação disponível no tempo $t$. Geralmente se faz a hipótese simplificadora de que as informações disponíveis no tempo $t$ (o conjunto de informação) são idênticas para todos os indivíduos nessa economia. Assim, podemos falar da esperança matemática condicional ao conjunto de informação no tempo $t$. Definimos $E\left[y_{t+1} \mid t\right]$ por

$$
E\left[y_{t+1} \mid \mathbf{t}\right]=E\left[y_{t+1} \mid \mathbf{I}_{t}\right]
$$

12 baseado no livro de Blanchard e Fisher (1989). 
onde

$$
I_{t}=\left\{y_{t-i}, x_{t-i}, z_{t-i}, i=1,2, \ldots, \infty\right\}
$$

$E\left[y_{t+1} \mid t\right]$ é igual à esperança matemática de $y_{t+1}$ condicional ao conjunto de informação $I_{t}$. O conjunto de informação contém os valores correntes e defasados das variáveis $y$ e $x$ e de outras variáveis incluídas no vetor $z_{t}$ que, embora não apareçam diretamente na equação (41), podem ajudar a predizer os valores de $y$ e $x$.

\section{Outros conceitos de equilíbrio e alguns resultados adicio- nais.}

Alguns economistas consideram que o EER impõe muitas restrições sobre o conhecimento que os agentes possuem sobre a economia. Um conceito de equilíbrio alternativo comumente usado e que impõe menos restrições sobre o conhecimento dos agentes é o conceito de equilıbrio temporário, conceito também introduzido por Hicks e desenvolvido sistematicamente em Grandmont (1974) e Stigum $(1969,1972)$, com uma excelente resenha em Grandmont (1982). Este conceito de equilíbrio só faz sentido em economias com mercados intertemporalmente incompletos, isto é, no período $t$ não existem mercados futuros completos para os bens nos períodos posteriores a $t$. Um equilíbrio temporário pode ser descrito como: dadas as crenças dos indivíduos, eles agem no mercado spot e nos mercados futuros existentes; todavia não se requer que as crenças dos indivíduos sejam consistentes entre si, nem com a verdadeira distribuição de probabilidade dos diversos estados da natureza nos períodos seguintes.

Um conceito de equilíbrio muito relacionado com o de EER é o de equilíbrio com manchas solares ou equilíbrio com incerteza extrínseca. ${ }^{13} \mathrm{~A}$ diferença básica entre os dois conceitos de equilíbrio é que enquanto no EER a incerteza é gerada por fundamentais ${ }^{14} \mathrm{da}$ economia, no equilíbrio com manchas solares a incerteza é gerada "na cabeça das pessoas". Os agentes acreditam que uma variável

\footnotetext{
${ }^{13}$ Esta é uma literatura muito grande atualmente. Referências seminais são Azariadis (1981), Shell (1977), Cass and Shell (1983) e uma recente resenha é Chiappori e Guesnerie (1991).

${ }^{14}$ Por fundamentais entendemos as variáveis que afetam a estrutura da economia como dotaçōes iniciais, tecnologia, preferências.
} 
aleatória tenha influĉncia sobre a economia, como por exemplo a ocorrência ou não de manchas solares. Embora manchas solares não afetem dotações preferenciais e tecnologias, (não estamos aqui fazendo um juízo sobre se de fato manchas solares afetam ou não os fundamentais da economia. Manchas solares aqui significam qualquer variável aleatória que não afete os fundamentais da economia) elas passam a afetar os preços e possivelmente as alocações finais, simplesmente porque os agentes crêem que tais manchas afetem algumas variáveis relevantes para suas decisões econômicas, como por exemplo os preços. As definições de equilíbrio são as mesmas nos dois modelos, a diferença entre eles é que em EER os estados da natureza são exógenos, enquanto que em equilíbrio com manchas solares os estados da natureza são gerados endogenamente.

Utilizando-se de métodos mais avançados de matemática, Allen (1981) e Radner (1979) mostram, num contexto de um contínuo de estados da natureza, que existe genericamente EER que revela toda a informação da economia se a dimensão do espaço das informações privadas for menor que a dimensão do espaço dos preços.

Kreps (1977) mostra que nem sempre pode-se garantir a existência de EER. Kreps constrói uma economia com dois tipos de agentes: informados e não-informados. A não-existência de EER é devido à seguinte característica do modelo. Suponhamos que exista equilíbrio e que os preços de equilíbrio revelem a informação dos informados para os não-informados. Neste caso todos ficam igualmente informados. Mas pelos dados do problema, se todos são igualmente informados, os preços de equilíbrio não revelam nenhuma informação. Logo, o equilíbrio não pode existir.

Anderson e Sonnenchein $(1982,1983)$, desenvolvem modelos de existência de equilíbrios com expectativas racionais, modificando um pouco o conceito de EER. Nos modelos de Anderson e Sonnenchein os agentes estão incertos sobre as relações entre suas informações privadas e as variáveis da estrutura da econornia, mas possuem uma classe de modelos dentro da qual acredita que um deles é o verdadeiro modelo da relação entre suas informações e as variáveis da economia. Um EER é um vetor de morlelos (um para cada agente), tal que se os agentes acreditam neles então depois de observarem a realização 
de mais informaçōes eles nĩo murdam de modelo.

Um outro problema muito importante ć o da convergência para equilíbrio com expectativas racionais. Sc os agentes da economia comesam com expectativas quaisquer, eventualmente convergiriam cles para expectativas racionais? Bray (1982) descreve um modelo muito interessante onde estimaşão por mínimos quadrados leva ao EER. Blume e Easley (1982) estudam o sistema dinâmico das expectativas geradas por um modelo simples de equilíbrio geral sobre o qual os agentes consideram um número finito de modelus. Cada modelo especifica uma relaşão entre informação e preços de equilíbrio. Um desses modelos é uma correta descrição de um EER. Como os agentes não sabem qual é o modelo correto de equilíbrio, cles usam uma média ponderada desses modelos. Eles mostram que se os agentes comeşam "perto" do equilíbrio com expectativas racionais (colocando peso alto para o modelo EER), cles eventualmente convergirão para ele. Todavia, se os agentes comeşarem longe do ELR pode ocorrer que cles convirjam para um equilíbrio que não é EER. Mais recentemente Araújo c Sandroni (1991) mostram que sc os agentes concordam sobre os eventos com probabilidade zero, comesando com distribuições a priori Guassianas arbitrárias, eles eventualmente convergirão para expectativas racionais; começando com distribuições a priori arbitrárias eles convergem para expectativas racionais com probabilidade positiva.

Finalmente, uma literatura mais recente tenta, estabelecer uma relaşão entre EER e tcoria dos jogos ${ }^{15}$. O EER deixa de ser um problema de inferência estatística de informação e passa a ser um problema onde os agentes passam a ter comportamento estratígico.

Submetido em junlıo de 1992. Revisado em março de 199.3.

\section{Referências}

Allen, B. 1981. "Generic existence of completely revealing equilibria for economies with uncertaint.y when prices convey information." Econometrica 49: 1173-99.

\footnotetext{
${ }^{15}$ Evans (1985), Guesnerie (1992), Simonsen (1986, 1988), Townsend (1978), entre outros.
} 
Anderson, R. \& Sonnenchein, H. 1982. "On the existence of rational expectations equilibrium." Journal of Economic Theory 26:261-278. 1985. "Rational expectations equilibrium with econometric models." Review of Economic Studies 52:359-369.

Araújo, A. \& Sandroni, A. 1991. "On convergence of bayesian priors to rational expectations in complete markets." Mimeo, IMPA, Rio de Janeiro.

Azariadis, C. 1981. "Self-fulfilling prophecies." Journal of Economic Theory 25: 380-396.

Blanchard, O. \& Fisher, S. 1989. Lectures on Macroeconomics. Cambridge, Mass: Mit Press.

Blume, L. E., \& Easley, D. 1982. "Learning to be rational." Journal of Economic Theory 26:340-351.

Bray, M. 1982. "Learning estimation and the stability of rational expectations." Journal of Economic Theory 26:318-39.

Cass, D. \& Shell, K. 1983. "Do sunspots matter?" Journal of Political Economy 91: 193-227.

Chiappori, P.\& Guesnerie, R. 1991. "Sunspot equilibria in seqüential market models." In Hildenbrand, M.L., \& Sonnenchein, H., eds., Handbook of Mathematical Economics IV. Amsterdam: North Holland.

Evans, G. 1985. "Expectational stability and multiple equilibrium problem in linear rational expectations models." Quartely Journal Economics 100:1217-33.

Guesnerie, R. 1992. "An exploration of the eductive justifications for the rational-expectations hypothesis." American Economic Review 85: 1254-78.

Grandmont, J. 1974. "On the short-run equilibrium in a monetary economy." In Dreze, J., ed., Allocation under Uncertainty, Equilibrium, and Optimality. London: MacMillan.

1982. "Temporary general equilibrium theory." In Arrow, K. \& Intrigator, M., eds., Handbook of Mathematical Economics volume II. Amsterdam: North-Holland.

Grossman, S. 1975. "Rational expectations and the econometric modeling of markets subject to uncertainty." Journal of Econometrics 3: 255-272. 
1976. "On the efficiency of competitive stock markets where traders have diverse information." Journal of Finance 31: $573-585$.

1977. "The existence of futures markets, noisy rational expectations and informational externalities." Review of Economic Studies 44: 431-449.

1978. "Further results on the informational efficiency of competitive stock markets." Journal of Economic Theory 18: $81-101$.

1979. "Rational expectation and the theory of resources allocation under asymmetric information: a survey.." CARESS Working Paper no 79-18, University of Pennsylvania.

1981. "An introduction to the theory of rational expectations under assimetric information." The Review of Econometric Studies 48:541-560.

Hayek, F. H. 1945. "The use of knowledge in society." American Economic Review 35:519-530.

Hicks, J. 1939. Value and Capital. London: Oxford University Press. Kreps, D. 1977. "A note on 'fulfilled expectations' equilibria." Journal of Economics Theory 14:32-43.

Lucas, R. 1972. "Expectations and the neutrality of money." Journal of Economics Theory 4:103-124.

Muth, J. R. 1960. "Optimal properties of exponentially weighted forecasts." Journal of the American Statistical Association 55:229305.

1961. "Rational expectations and the theory of price movements." Econometrica 24:315-335.

Nerlove, M. 1958. The Dynamics of Supply: Estimation of Farmers' Response to Price. Baltimore: John Hopkins Press.

Radner, R. 1979. "Rational expectations equilibrium: generic existence and the information revealedd by price." Econometrica 47: $655-78$.

Shell, K. 1977. "Monnaie et allocation intertemporelle." Mimeo, Séminaire d'Econométrie. Paris: Roy-Malinvaud.

Simonsen, M. 1986. "Rational expectations income policies and game theory." Revista de Econometria 6:7-46. 
1988. "Rational expectations, game theory and inflacionary inertia." In Arrow, K., ed., The Econorny as an Evolving Complex, System,. New York: McGraw Ifill.

Stigum B. 1969. "Competitive equilibria under uncertainty." Quarterly Journal of Econornics 83:533-561.

1974. "Competitive resource allocation over time under uncertainty." In Balch, M., McFadden, D. \& Yu, S., cds., Essays on Econornic Behavior under Uncertainty, Contribulions to Econornic Analysis. Amsterdam: North-Holland.

Townsend, R. 1978. "Market antecipations, rational expectations and bayesian analysis." Inlernational Economic Review 19: 481-94. 